\title{
Hyperfine anomalies in the ammonia $(1,1)$ inversion transition: Can they be a tracer of systematic motion?
}

\author{
Y.-S. Park ${ }^{\star}$ \\ Astronomy Program, SEES, Seoul National University, Shillim-dong Kwanak-gu, Seoul 151-742, Korea
}

Received 3 January 2001 / Accepted 27 June 2001

\begin{abstract}
The hyperfine lines of an ammonia $(1,1)$ inversion transition often exhibit anomalous intensity ratios towards star-forming cores. It has been known that the anomaly is only partially attributable to a non-LTE effect on the hyperfine transitions. In an attempt to investigate how systematic motion like expansion and contraction affect the line anomaly, whose existence is quite likely in star-forming cores, we have carried out radiative transfer calculations fully taking into account the hyperfine splitting of energy levels and the line overlap effect caused by the systematic motion. It is demonstrated that in a bundle of transitions from the $(2,2)$ to $(1,1)$ levels, photons emitted from one transition can be absorbed by another transition due to the systematic motion and the small frequency differences among the hyperfine transitions, resulting in drastic changes in the level populations of $(1,1)$ sub-states. Expansion (contraction) is found to strengthen the inner as well as outer satellite lines on the red (blue) side, while suppressing those on the other side. The line anomaly becomes prominent as the ammonia column density increases. It is concluded that the hyperfine line intensity ratios could be a good probe for tracing the systematic motion inside the dense cores without relying on detailed analysis of line shapes.
\end{abstract}

Key words. ISM: clouds - ISM: kinematics and dynamics - ISM: molecules - line: formation - radiative transfer

\section{Introduction}

Ammonia inversion lines have been widely used as a thermometer or as a density tracer (Ho \& Townes 1983; Benson \& Myers 1989; Lemme et al. 1996; Jijina et al. 1999). The ammonia (1,1) inversion line, which is the most commonly observed, has two hyperfine lines on each side of the main line. If they are optically thin, the inner (outer) satellite lines are expected to have $0.26(0.22)$ times the intensity of the main line, and if they are opaque, their line intensities are all equal. However, an interesting phenomenon called the "hyperfine anomaly" has long been known and a thorough investigation has been undertaken to show that the outer satellite line on the red side, that is stronger than the one on the blue side, results from the non-LTE level populations due to the hyperfine selective photon trapping (Stutzki \& Winnewisser 1985b, SW85 hereafter).

However, observations exhibit a wider range of anomalies, as shown, e.g., in Anglada et al. (1997). Our work is actually motivated by the VLA observation of a dense core embedding a young stellar object (YSO), IRAS $19550+3248$, toward which the inner satellite line on the low velocity side is found to be very weak
(Lee et al., in preparation). These cannot be explained simply by the non-LTE effect, because according to SW85, the inner satellite lines are insensitive to it and their strength does not fall below the optically thin limit, 0.26 of the main line strength. A survey of the literature revealed a similar feature in the outflow lobe of L1157 (Umemoto et al. 1999). Since the material embedded in these YSOs involves a variety of motions such as infall and outflow, the velocity field may play a role in producing the anomaly. Since the ammonia hyperfine transitions are located so close together in frequency, a photon emitted from one transition might be absorbed in another transition via the Doppler shift. DR21 is a proto-typical example of this type of anomaly (Matsakis et al. 1977; Gaume et al. 1996). Though it is somewhat complicated by the continuum emission, the authors suggest that the systematic motion is responsible for the anomalous hyperfine lines. Matsakis et al. (1977) conducted numerical calculations, but their treatment was only qualitative. Thus an attempt is made here to better understand the peculiar behavior of the hyperfine lines, with an emphasis on the role of the systematic motion inside the ammonia core unexplored by SW85.

* e-mail: yspark@astro.snu.ac.kr 


\section{Models}

We made use of the Monte Carlo radiative transfer code developed by Bernes (1979) and modified it to incorporate the line overlap effect (Park et al. 1999; Lapinov 1989). The core is gridded into 15 concentric shells whose radii vary as $r_{i}=R(i / 15)^{0.4}, i=0,1,2, \ldots, 15$. Since we are primarily concerned with ammonia cores associated with low-mass star formation, we adopt a radius of $R=$ $0.04 \mathrm{pc}$ and a constant kinetic temperature of $T=15 \mathrm{~K}$, based on both single dish and interferometer observations toward the cores (Zhou et al. 1989; Jijina et al. 1999; Fuller \& Wooten 2000; Lee et al., in preparation). It may be appropriate to introduce density profiles decreasing outward, but in order to make the problem as simple as possible, the density distribution is assumed to be uniform.

The role of the velocity field in determining the anomalous line intensity should be understood in relation to the hyperfine structure of ammonia energy levels, which is best illustrated in Fig. 1 of SW85. Considering the frequency differences among the hyperfine lines of $(2,1) \rightarrow(1,1)$ transitions that govern the populations of $(1,1)$ sub-states, the systematic gas motion should be $\gtrsim 0.3 \mathrm{~km} \mathrm{~s}^{-1}$ in order for the line overlap to be at work. Yet observations suggest that it should not be too large, $\lesssim 0.5 \mathrm{~km} \mathrm{~s}^{-1}$ (Choi et al. 1995; Gregersen et al. 2000). For the velocity law of systematic motion, one can think of either free fall of a gas sphere $(v(r) \propto r)$, or inside-out collapse $\left(v(r) \propto r^{-0.5}\right)$. An important point in examining the line overlap effect is whether any photons and if so, how many photons from a transition are absorbed by another transition. That is, of crucial importance is the sense and magnitude of the photon Doppler shift as seen by an ammonia molecule in a certain position of the core. In the case of uniform contraction, the incident photons are all blue-shifted, independent of the position of the ammonia. The Doppler shift is rather isotropic, but not perfectly, of course, because of the finite size of the core. On the other hand, for inside-out collapse, this may not be the case, since in a certain direction, i.e., toward the center or radially outward, incident photons are red-shifted in contrast to an overall blue shift in the other direction. The photons from a half sphere on the other side of the core center as seen from an observer is always blue-shifted, independent of the velocity law. Part of a half sphere on the observer's side also generates blue-shifted photons, the fraction depending on the position of the ammonia that receives photons. Thus the volume from which photons are blue-shifted is much larger than that from which photons are red-shifted, the volume fraction depending on the ammonia position. The relative line intensity would not be much affected by the adopted velocity law, although the line shape might. Thus we decided to use the model of uniform contraction as a representative law of the internal motion. For comparison, it will be useful to explore the opposite case of outward motion as well. To summarize, we adopt the velocity model of $v(r)= \pm V_{0}(r / R)$, where the plus and minus signs refer to the outward and inward motion, respectively. Most calculations are undertaken with $V_{0}=0.4 \mathrm{~km} \mathrm{~s}^{-1}$ obeying the constraints mentioned above, but models with different values of $V_{0}$ are also explored and discussed in Sect. 3.4.

On the other hand, micro-turbulence motion tends to weaken the selective line overlap effect. Thus, in order to discern the line overlap effect, we assume only pure thermal motion for the Gaussian line absorption profile, i.e., $\Delta V_{F W H M}=0.2 \mathrm{~km} \mathrm{~s}^{-1}$ corresponding to a kinetic temperature of $15 \mathrm{~K}$. If the turbulence plus thermal motion is larger than $\approx 0.6 \mathrm{~km} \mathrm{~s}^{-1}$, all the lines from $(2,1) \rightarrow(1,1)$ will be merged into one, resulting in a weak line anomaly of $(1,1)$ inversion transitions. The effect of non-thermal microturbulence is also investigated (see Sect. 3.5).

Then we are left with two major free parameters, the hydrogen number density, $n\left(\mathrm{H}_{2}\right)$, and the abundance of ammonia, $X\left(\mathrm{NH}_{3}\right)$ or $n\left(\mathrm{NH}_{3}\right) / n\left(\mathrm{H}_{2}\right)$. We explored the parameter spaces of $n\left(\mathrm{H}_{2}\right)=1 \times 10^{4} \mathrm{~cm}^{-3}$ to $1 \times 10^{7} \mathrm{~cm}^{-3}$ and of $X\left(\mathrm{NH}_{3}\right)=1 \times 10^{-9}$ to $3 \times 10^{-7}$. The lower boundary of $X\left(\mathrm{NH}_{3}\right)$ is based both on the observation by Fuller \& Wooten (2000) and on the gas-grain chemical calculation of Shalabiea (2001). However, we excluded cases for which the main line of the $(1,1)$ inversion transition is too optically thick $\left(\tau_{\mathrm{M}} \gtrsim 10\right)$ or the line is too weak $\left(T_{\mathrm{B}} \lesssim 1 \mathrm{~K}\right)$.

Statistical equilibrium equations are solved with 30 hyperfine levels of $(J, K)=(1,1),(2,1),(3,1),(2,2)$, and $(3,2)$ and 70 radiative transitions among them. Stutzki $(2000$, private communication) provided us with energy level and Einstein coefficients. Hyperfine selective collisional coefficients are taken from Stutzki \& Winnewisser (1985a). It is found that the code converges slowly particularly in the optically thick cases, and that a large number of photons $\left(\sim 10^{5}\right)$ are necessary for safe convergence. The code is iterated until the level populations of the lowest 12 levels change by no more than $0.3 \%$ for about 5 consecutive iterations. Sometimes the transitions become suprathermal, making the calculations unstable. However, repeated calculations with different seed numbers ensure that the resulting line profiles are accurate to a few percent.

\section{Results}

\subsection{Comparison with SW85}

At first, for comparison, we carried out calculations for models similar to the ones considered by SW85. Model parameters are $T=25 \mathrm{~K}, n\left(\mathrm{H}_{2}\right)=3 \times 10^{5} \mathrm{~cm}^{-3}$, and $R=0.02$ pc. We choose the $T=25 \mathrm{~K}$ model, since the line anomaly is larger for the model of higher temperature. Figure 1 shows the line profiles from the center of the core and those averaged over the disk, the projection of the core onto the sky plane. The latter profiles can be compared with the second row of Fig. 4 in SW85. In the figure, the column density divided by the line width, $10^{14.6}-$ $10^{15.8} \mathrm{~cm}^{-2} \mathrm{~s} \mathrm{~km}^{-1}$, corresponds to an $\mathrm{NH}_{3}$ abundance 


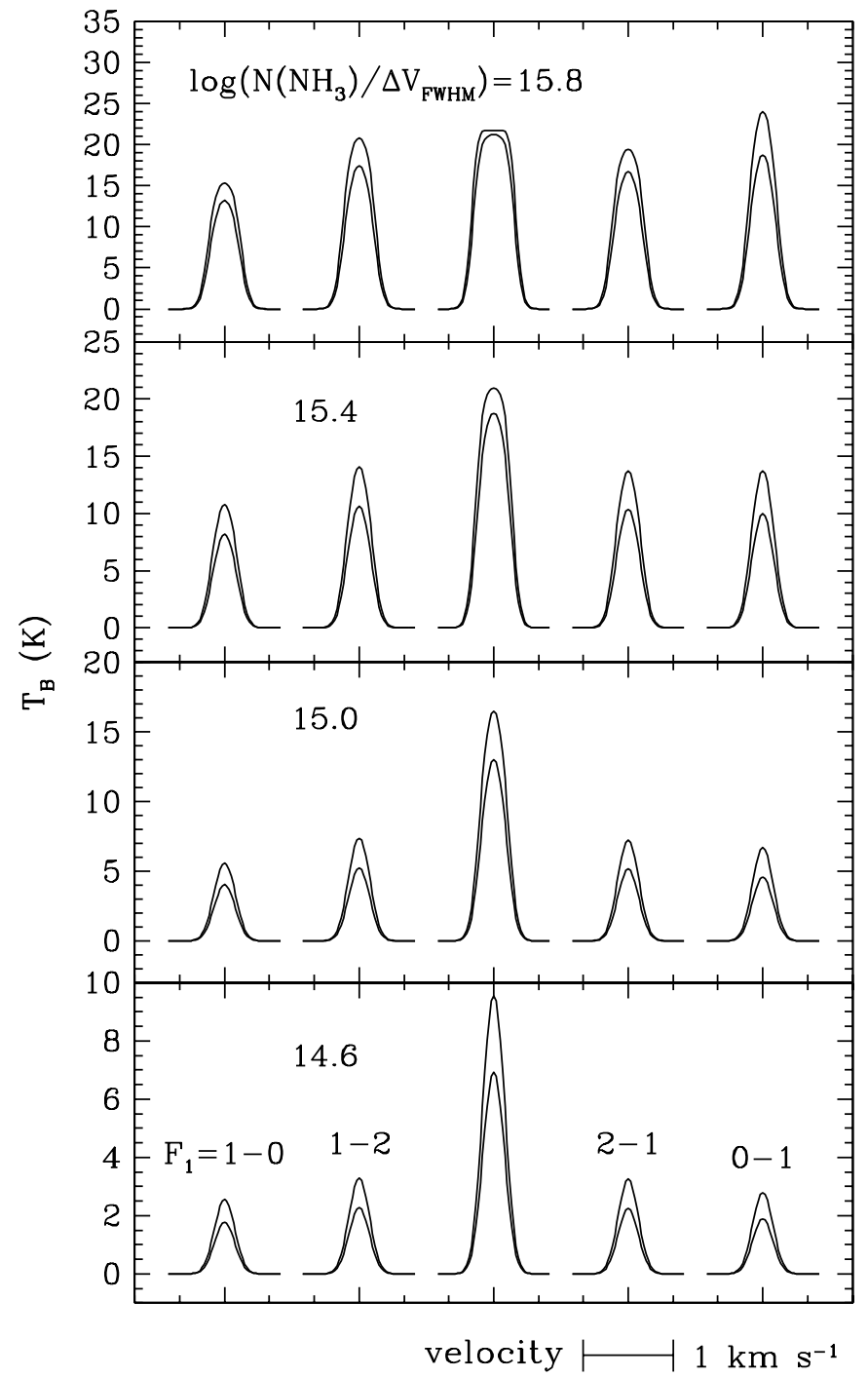

Fig. 1. Profiles of ammonia $(1,1)$ inversion lines from the static cores of various column densities, where $n\left(\mathrm{H}_{2}\right)=3 \times 10^{5} \mathrm{~cm}^{-3}$, $T=25 \mathrm{~K}$, and $R=0.02 \mathrm{pc}$. The upper curves are line emissions from the center of the core and the lower ones are the profiles averaged over the core in the plane of the sky. The number in the top left corner of each figure frame represents column density per unit velocity. The velocity scale is shown at the bottom.

relative to molecular hydrogen of $3.1 \times 10^{-9}-4.9 \times 10^{-8}$ for a pure thermal width of $\Delta V_{F W H M}=0.26 \mathrm{~km} \mathrm{~s}^{-1}$.

It is found that the lines are generally weaker and the line anomalies of outer satellite lines are less prominent than those of SW85. One should be aware that SW85 applied the escape probability method and assumed uniform excitation conditions over all depth points which are equal to those at the center of the core. Using the smallest value of escape probability at the center leads to a tendency towards greater ammonia population in higher energy levels. Since we relax this restriction, the lines calculated in this work are generally fainter. Moreover, the anomaly will be weaker in the outer part of the core than that found by SW85, and consequently the lines averaged over the core are less anomalous. They also assumed to be an "all or nothing" line overlap whereby transitions within a thermal line width are considered to have an identical frequency, whereas those further apart from each other are completely separated. By contrast, we deal with the problem without employing this assumption at the cost of increased computation time. However, our calculation shows that this simplification does not alter the results too much.

\subsection{Static cores}

Coming back to the model of $T=15 \mathrm{~K}$, we conducted the calculations for three kinds of models: static, expanding, and contracting cores. From now on we will refer to the line emission toward the core center only. The resulting line profiles from the static cores are shown together with those from the cores with inward and outward motion in Figs. 2 and 3, respectively, where the ammonia abundance of the top left frame is $X\left(\mathrm{NH}_{3}\right)=3 \times 10^{-7}$, and that of the rightmost frames in each row is $X\left(\mathrm{NH}_{3}\right)=1 \times 10^{-9}$. At low column density, the lines from the static cores are brighter than those of the cores with motion simply due to low velocity dispersion. The line intensities are most sensitive to the ammonia column density. They also slowly increase along with the $n\left(\mathrm{H}_{2}\right)$, and will eventually reach the limiting brightness of $T_{\mathrm{B}} \simeq 12 \mathrm{~K}$. It is found that the line anomaly is the most prominent for a column density of $N\left(\mathrm{NH}_{3}\right) \sim 3 \times 10^{14} \mathrm{~cm}^{-2}$ or for a main line optical depth of $\tau_{\mathrm{M}} \sim 3$; however, as a whole, it is not as remarkable as that of the high temperature models.

\subsection{Cores under systematic motion}

Figures 2 and 3 also present the line profiles in the presence of the systematic motion. Like the case of the static cores, the anomaly largely depends on the ammonia column density. At low column density, the line seems normal, and the anomaly begins to develop for $N\left(\mathrm{NH}_{3}\right) \sim$ $3 \times 10^{14} \mathrm{~cm}^{-2}$. The corresponding main line optical depth is around unity, suggesting that the anomaly takes place at a lower optical depth in comparison with the static cores. The anomaly is much stronger than that of the static model and, as the column density increases further, satellite lines become even stronger than the main line. It should be noted that for the cores with the systematic motion, inner satellite lines also become anomalous, unlike the static cores. For the largest column density, the lines are thermalized as $n\left(\mathrm{H}_{2}\right)$ increases, since the collisional process dominates the radiative one. The sense of anomaly is reversed when the direction of motion is reversed, i.e., the brightest line is on the blue side of the contracting cores, while it is on the red side of the expanding cores with similar line strength.

The anomaly can be explained in terms of photon exchange. The reader is referred to Fig. 1 of SW85 for the energy level diagram of ammonia. In a core collapsing 




Fig. 2. Profiles of ammonia $(1,1)$ inversion lines are shown from the center of the static (narrow line profiles) and contracting (broad ones) cores for various ammonia column densities and hydrogen number densities, where $T=15 \mathrm{~K}$ and $R=0.04 \mathrm{pc}$. The velocity scale is shown at the bottom.

uniformly $(v(r) \propto r)$, all of the photons incident on an ammonia molecule in a depth point is blue-shifted and the degree of blue shift is proportional to the distance to the position where the photon is last emitted. Thus the photons from the $(2,1) \rightarrow(1,1)$ main transitions $\left(F_{1}=3-2\right)$ can be absorbed in the satellite transitions of $F_{1}=1-0$, while the main line transitions receive photons from the $F_{1}=2-1$ transitions. The $F_{1}=2-1$ transitions have only to emit photons without any return. These result in overpopulation of the $F_{1}=1$ states of $(1,1)$ levels and underpopulation of the $F_{1}=0$ states. Consequently, the $F_{1}=1-$ 0 line of the $(1,1)$ inversion transition gets stronger, while the $F_{1}=0-1$ becomes weaker. Those photon trapping processes are more efficient for larger optical depth. For even higher column density, the $F_{1}=1-0$ transition is inverted. In the case of outward motion, the opposite explanation applies. As mentioned in Sect. 2, for a different velocity law such as the inside-out collapse $\left(v(r) \propto r^{-0.5}\right)$, a certain fraction of photons is red-shifted. However, additional calculations show that the conclusion drawn here is generally valid, since the fraction is small.

Considering the velocity law of $V(r)= \pm 0.4$ $(r / R) \mathrm{km} \mathrm{s}^{-1}$ and the velocity differences, $\lesssim 0.3 \mathrm{~km} \mathrm{~s}^{-1}$ of $(2,1) \rightarrow(1,1)$ transitions, the absorption of Doppler-shifted photons can be most effective in the central region. An ammonia molecule in the outer boundary can absorb blue- or red-shifted photons only from a half shell centered at the molecule's position touching the core center. Thus the differences in the excitation temperatures among hyperfine transitions will be larger in the core center than at the core edge. For the velocity law of uniform contraction or expansion, the excitation temperature along a radial distance has a one-to-one correspondence with the intensity across the line profile if the transitions are optically thick. Thus, the strong hyperfine line on the blue (red) side of collapsing (expanding) cores with the largest column density is a manifestation of suprathermal excitation of the transition around the core center, while the weak line with the line core suppressed on the other side represents a subthermal excitation.

\subsection{Changing the amplitude of velocity}

We calculated line emissions by varying $V_{0}$ for a model core with the fixed values of $n\left(\mathrm{H}_{2}\right)=3 \times 10^{5} \mathrm{~cm}^{-3}$ and $X\left(\mathrm{NH}_{3}\right)=3 \times 10^{-8}$, and those are displayed in Fig. 4 . With increasing $V_{0}$ the lines become weaker, particularly for the transitions that are formerly suprathermally excited. In the presence of a large velocity gradient, the volume of a shell from which an ammonia in a certain position receives Doppler-shifted photons becomes less depth dependent. Then the excitation condition will also have little positional dependence, resulting in a line profile with 


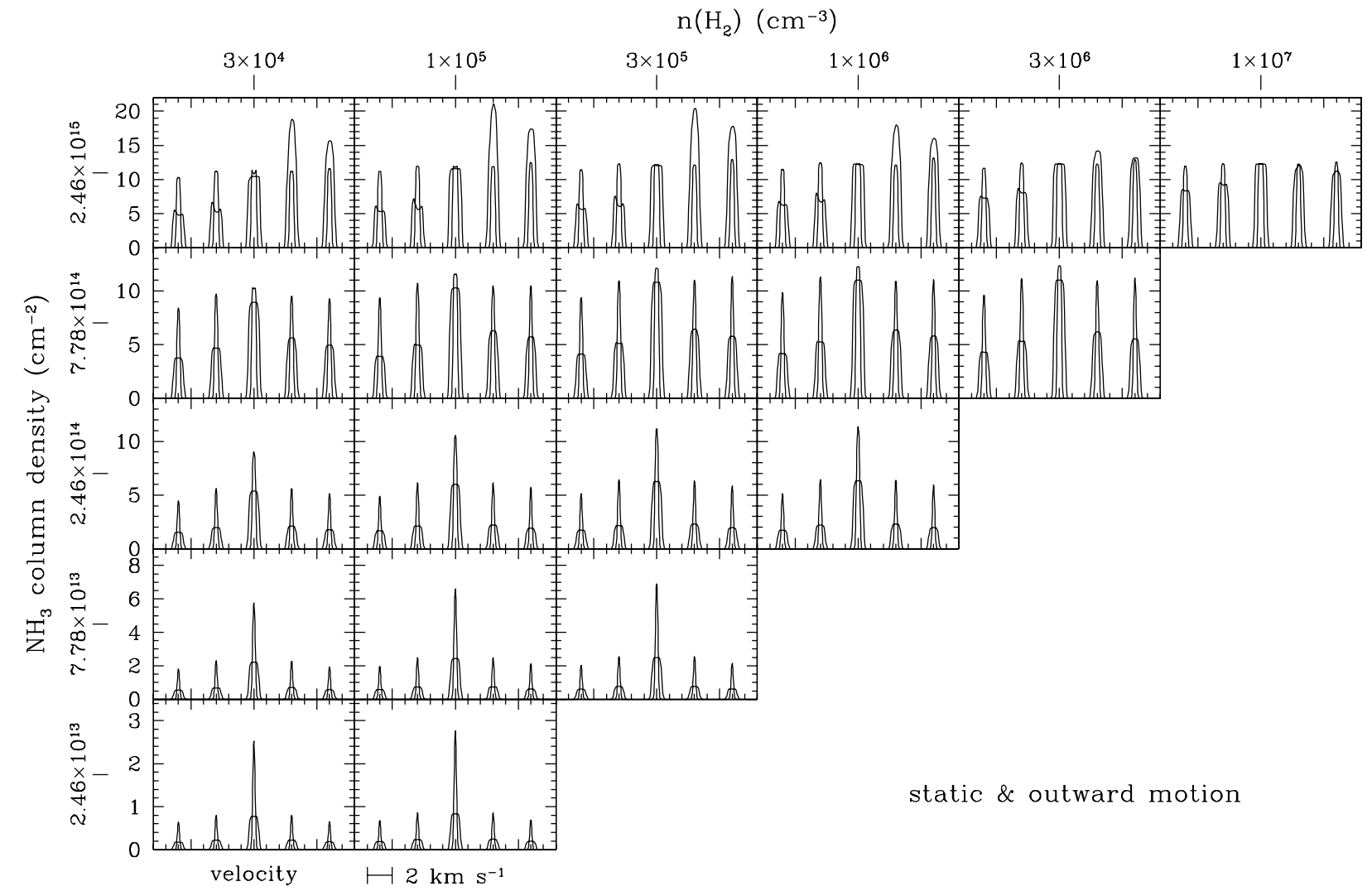

Fig. 3. Profiles of ammonia $(1,1)$ inversion lines are shown from the center of the cores with expansion (broad line profiles) and of the static cores (narrow ones) for various ammonia column densities and hydrogen number densities, where $T=15 \mathrm{~K}$ and $R=0.04 \mathrm{pc}$. The velocity scale is shown at the bottom.

a flat top for the uniform density distribution. It is found that the anomaly keeps appearing, since the photon exchanging process continues to work. It will vanish only when the velocity gradient is so large that the excitation condition becomes a purely local one. This contrasts with the case of microturbulence.

\subsection{Changing the turbulence velocity}

One can easily understand that the photon exchange will become less important as the turbulent velocity increases. If the velocity is larger than the line separation of a bundle of $(2,1) \rightarrow(1,1)$ transitions, then the transitions will behave as a single line and the $(1,1)$ inversion lines will no longer exhibit the anomaly. In order to confirm this reasoning, additional calculations were conducted and the resulting line profiles are displayed in Fig. 5. The figure shows that the anomaly decreases with increasing turbulent velocity, $v_{\text {turb }}$. For $v_{\text {turb }} \approx 0.6 \mathrm{~km} \mathrm{~s}^{-1}$, the inner or outer satellite lines of both sides are almost equal in strength.

\section{Discussions}

\subsection{Applicability of the model}

One of the drawbacks of this work is that the synthesized line is generally brighter than the observed one, a deficiency which is shared by all previous studies assuming a smoothly varying gas distribution. For this reason, a clumpy core model has been invoked in which a large number of small clumps partially fill the telescope beam (e.g., SW85). For the objects like L1251A, L483, and L1262 exhibiting prominent ammonia anomalies (e.g., Anglada et al. 1997; see next section), the main beam brightness of the main line components is found to be $T_{\mathrm{MB}} \approx 1.5-4 \mathrm{~K}$, whereas the brightness temperature of the synthesized line is $T_{\mathrm{B}} \approx 7-12 \mathrm{~K}$ for the excitation temperature of $T_{\mathrm{ex}} \approx T_{k} \approx 10-15 \mathrm{~K}$, since the main line optical depth is $\tau_{\mathrm{M}} \gtrsim 1$. Taking into account the fact that the observed ammonia core is marginally resolved by telescope beam and the line emission that we are dealing with is from the core center, the area filling factor of clumps is estimated to be $\gtrsim 0.3$. Thus it would be plausible to incorporate the clumpy picture into the core in which clumps only with thermal motion take part in the global expansion or collapse of the core. The hyperfine anomaly may become weaker due to clumpiness, since the number of Doppler-shifted photons of $(2,1) \rightarrow(1,1)$ transitions decreases. This could be compensated by increasing ammonia abundance and/or gas density. Considering the area filling factor, one may find that the area-averaged ammonia column density changes little to produce a similar degree of hyperfine anomaly (Park \& Hong 1995). Since the anomalous $(1,1)$ inversion lines are rather opaque, 


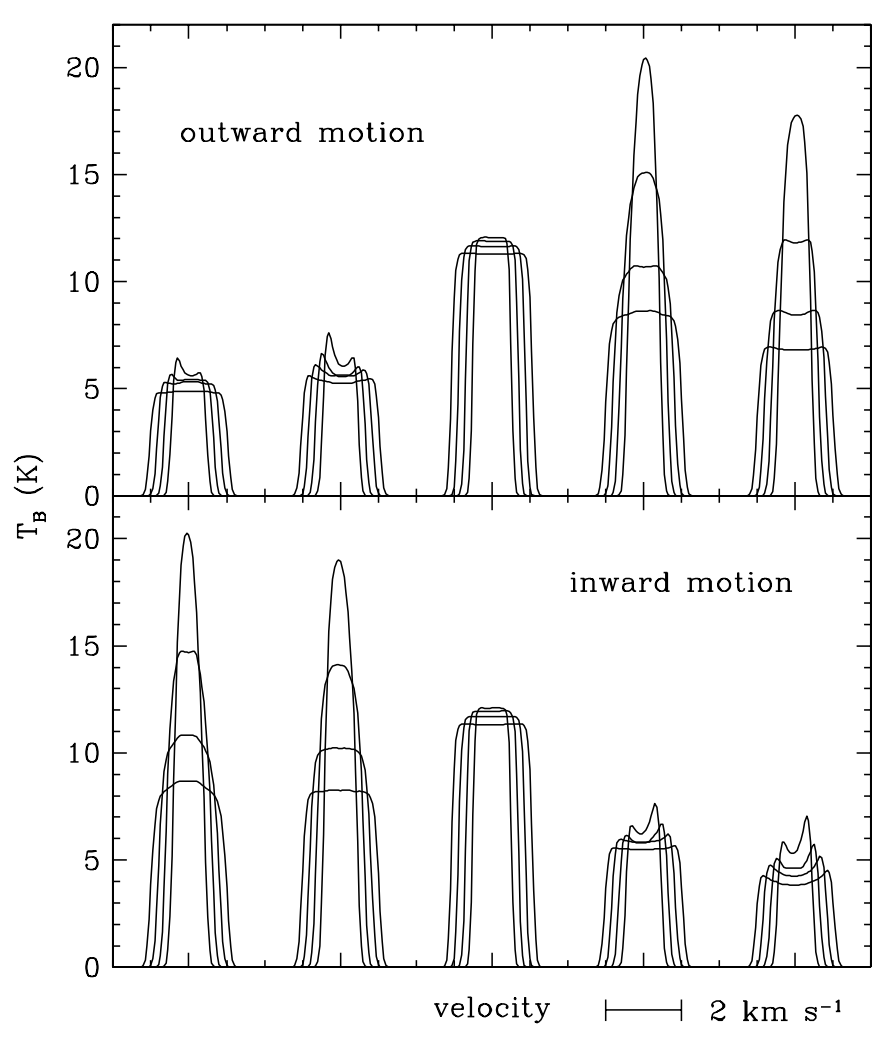

Fig. 4. Profiles of ammonia $(1,1)$ inversion lines from the center of the cores for several $V_{0}$ 's of $V(r)= \pm V_{0}(r / R)$. The $V_{0}$ changes from $0.4 \mathrm{~km} \mathrm{~s}^{-1}$ (narrowest line profile) to $1.0 \mathrm{~km} \mathrm{~s}^{-1}$ (widest one) with an increment of $0.2 \mathrm{~km} \mathrm{~s}^{-1}$. The $n\left(\mathrm{H}_{2}\right)$ and $X\left(\mathrm{NH}_{3}\right)$ are fixed to $3 \times 10^{5} \mathrm{~cm}^{-3}$ and $3 \times 10^{-8}$, respectively, where $T=15 \mathrm{~K}$ and $R=0.04 \mathrm{pc}$. The velocity scale is shown at the bottom.

their intensities are affected by the area filling factor. In this way, the hyperfine lines could be less bright, but retain their anomalies. This explanation is very qualitative and thus it is intriguing to carry out three-dimensional radiative transfer calculations in order to investigate in detail the effect of clumpiness on the excitation conditions and the resulting line emissions in these clumpy cores (Park \& Hong 1998; Juvela 1997).

Another constraint we set is that the turbulence should be minimal and only the systematic motion is allowed. As shown in Sect. 3.5 , a turbulence of $v_{\text {turb }} \approx 0.6 \mathrm{~km} \mathrm{~s}^{-1}$ causes the line anomaly to diminish. Then one may argue against neglecting the turbulence. The line broadening dominated mainly by thermal motion is easily found in the cold dense cores (Bourke et al. 1995; Lemme et al. 1996) and even in the molecular outflow regions (Anglada et al. 1997). Barranco and Goodman (1998) \& Goodman et al. (1998) clearly demonstrate that the line width of quiescent ammonia cores is close to the pure thermal one. Thus one can expect that the systematic motion can be detected in the cores, if at all. However, this condition may not be met in warm or hot dense cores associated with HII regions or related to high mass star-forming activity where various sources of (micro-)turbulence are likely to be present.

\subsection{Comparison with observations}

We find a variety of ammonia lines in the literature (Batrla et al. 1983; Stutzki et al. 1984; Lemme et al. 1996; Anglada et al. 1997; Zinchenko et al. 1997). In earlier observations toward the cores forming high mass stars, the lines are wide, $\gtrsim 1 \mathrm{kms}^{-1}$ in general (e.g., Batrla et al. 1983; Stutzki et al. 1984). For several objects, the inner satellite lines appear to have equal intensity but the outer satellite lines have unequal intensity in spite of their large line width, which led SW85 to investigate the non-LTE effect of ammonia molecules in the clumpy structured cores.

One can find the most quiescent ammonia cores in the study of Lemme et al. (1996), toward which the magnetic hyperfine splitting of $(1,1)$ quadrupole groups is observable (Rydbeck et al. 1977). The lines are generally normal, suggesting little sign of systematic motion inside the cores.

The ammonia lines from outflow sources are wider and appear to exhibit diverse anomalies particularly in the inner satellite lines (Anglada et al. 1997). When comparing the synthesized lines with observed ones that are not so wide, one should keep in mind the fact that the magnetic splitting is the smallest $(\approx 11 \mathrm{KHz})$ in the hyperfine lines of the lowest velocity and about the same as the larger values of $\approx 33-46 \mathrm{KHz}$ in the others (Rydbeck et al. 1977). In the case of L1251A embedding IRAS 22343+7501 (and possibly L673), the four satellite lines become weaker in succession from the blue to the red (see Fig. 1 of Anglada et al. 1997). The line width is $\approx 0.6 \mathrm{kms}^{-1}$. If we exclude the lowest velocity line by the reason mentioned above, their relative intensities are qualitatively consistent with those of a collapsing core with $N\left(\mathrm{NH}_{3}\right) \approx$ $6 \times 10^{14} \mathrm{~cm}^{-2}$. The estimated LTE optical depth of the main line, $\tau_{\mathrm{M}}=1.5$ is not much different from $\tau_{\mathrm{M}} \approx$ 2.0 derived from the model. In fact, L1251A has been observed in numerous transitions of molecules such as $\mathrm{CS}, \mathrm{H}_{2} \mathrm{CO}, \mathrm{HCO}^{+}$, and $\mathrm{HCN}$, but the sense of motion is controversial (Mardones et al. 1997; Park et al. 1999; Gregersen et al. 2000). Diverse velocity fields can coexist in YSOs including collapse in the disk/envelope and expanding motion in the outflow, and the sensitivity to a certain velocity field may differ from one molecular transition to another. Since L1251A has been studied mostly by single point observations, further (mapping) observations are necessary in various molecular transitions to clarify its kinematical properties.

Objects like L483 and L1262 (Anglada et al. 1997) with the inner satellite weaker on the blue side than the red, can only be explained by outward motion; neither a collapsing nor static core can account for it. The strength of the outer satellite line in the blue may be due to the magnetic hyperfine splitting. The ammonia lines in the 




Fig. 5. Profiles of ammonia $(1,1)$ inversion lines from the center of the cores for a range of microturbulences. The velocity dispersions are $v_{\text {turb }}=$ $0.0,0.15,0.45$, and $0.75 \mathrm{~km} \mathrm{~s}^{-1}$, from left to right, respectively. The $n\left(\mathrm{H}_{2}\right), X\left(\mathrm{NH}_{3}\right)$, and $V_{0}$ are $3 \times$ $10^{5} \mathrm{~cm}^{-3}, 3 \times 10^{-8}$, and $0.4 \mathrm{~km} \mathrm{~s}^{-1}$, respectively, where $T=15 \mathrm{~K}$ and $R=0.04 \mathrm{pc}$. The velocity scale is shown at the bottom. outflow lobe of L1157 are also consistent with the expanding model (Umemoto et al. 1999).

One extreme case of a weak blue side inner satellite line is found in IRAS $19550+3248$. Toward this source, the inner satellite line on the blue side is almost missing, while the one on the red side has 0.8 times the intensity of the main line. In general, the absence of a line suggests either an optically thick line with very low excitation temperature or an extremely optically thin one. None of our calculations produces such a big contrast in excitation temperature or line intensity between the inner satellite lines. This will be discussed elsewhere in greater detail (Lee et al., in preparation).

DR21 is an exceptional object in the sense that the outer satellite line on the red side appears in emission and masering, while all the other lines are manifested in absorption (Gaume et al. 1996). According to our calculation, the maser on the red side occurs when the optical depth of the main line is rather high $\left(\tau_{\mathrm{M}}>10\right)$ for an expanding core. However, the inner satellite line is more likely to be inverted than the outer satellite one. In order to simulate the absorption lines, we undertook calculations in which the background radiation of all the relevant transitions including the FIR transitions is gradually increased from $2.7 \mathrm{~K}$ to $17 \mathrm{~K}$. However, it was found that the inner satellite line at higher velocity is the last transition to be in absorption due to the higher excitation temperature. Thus we failed to reproduce the features of DR21 with this simple model, suggesting that more sophisticated modeling is needed.

As the comparison with observations is rather tentative, the study demonstrates that the anomaly of inner satellite lines is attributable to the internal systematic motion.

\section{Summary and conclusions}

There exist diverse motions such as outflow, infall, and/or rotation in the cores embedding YSOs. Our calculations show that the relative line strength of ammonia inversion transitions is sensitive to the velocity field of the cores. Most recent studies seeking infall signatures in the star-forming cores rely on the detailed analysis of line shape (e.g., Zhou 1995; Mardones et al. 1997). This inevitably requires observations of high spectral resolution and signal-to-noise ratio that can not be easily accomplished, particularly in interferometric observations. In this respect, the ammonia inversion lines could be a good velocity probe of the cores since the line intensities can be measured with lower $S / N$ and spectral resolution than the line shape.

Acknowledgements. The author is grateful to an anonymous referee for invaluable comments and criticisms and wishes to thank J. Stutzki for providing molecular data. This research was supported by grant No. 2000-1-11300-002-3 from the Basic Research Program of the Korea Science and Engineering Foundation.

\section{References}

Anglada, G., Sepúlveda, I., \& Gómez, J. F. 1997, A\&AS, 121, 255

Barranco, J. A., \& Goodman, A. A., 1998, ApJ, 504, 207

Batrla, W., Wilson, T. L., Bastien, P., \& Ruf, K. 1983, A\&A, 128,279 
Benson, P. J., \& Myers, P. C. 1989, ApJS, 71, 89

Bernes, C. 1979, A\&A, 73, 67

Bourke, T. L., Hyland, A. R., Robinson, G., James, S. D., \& Wright, C. M. 1995, MNRAS, 276, 1067

Choi, M., Evans, N. J. II, Gregersen, E. M., \& Wang, Y. 1995, ApJ, 448, 742

Fuller, G. A., \& Wooten, A. 2000, ApJ, 534, 854

Gaume, R. A., Wilson, T. L., \& Johnston, K. J. 1996, ApJ, 457, L47

Girart, J. M., Estalella, R., Anglada, G., et al. 1997, ApJ, 489, 734

Goodman, A. A., Barranco, J. A., Wilner, D. J., \& Heyer, M. H. 1998, ApJ, 504, 223

Gregersen, E. M., Evans, N. J. II, Mardones, D., \& Myers, P. C. 2000, ApJ, 533, 440

Juvela, M. 1997, A\&A, 322, 943

Ho, P. T. P., \& Townes, C. H. 1983, ARA\&A, 21, 239

Jijina, J., Myers, P. C., \& Adams, F. C. 1999, ApJS, 125, 161

Lapinov, A. V. 1989, Soviet Astron., 33, 132

Lemme, C., Wilson, T. L., Tieftrunk, A. R., \& Henkel, C. 1996, A\&A, 312, 585
Mardones, D., Myers, P. C., Tafalla, M., et al. 1997, ApJ, 489, 719

Matsakis, D. N., Brandshaft, D., Chui, M. F., et al. 1977, ApJ, 214, L67

Park, Y.-S., Kim, J., \& Minh, Y. C. 1999, ApJ, 520, 223

Park, Y.-S., \& Hong, S. S. 1995, A\&A, 300, 890

Park, Y.-S., \& Hong, S. S. 1998, ApJ, 494, 605

Rydbeck, O. E. H., Sume, Å., Hjalmarson, A., et al. 1977, ApJ, 215, L35

Shalabiea, O. M. 2001, A\&A, 370, 1044

Stutzki, J., Jackson, J. M., Olberg, M., Barrett, A. H., \& Winnewisser, G. 1984, A\&A, 139, 258

Stutzki, J., \& Winnewisser, G. 1985a, A\&A, 144, 1

Stutzki, J., \& Winnewisser, G. 1985b, A\&A, 144, 13 (SW85)

Umemoto, T., Mikami, H., Yamamoto, S., \& Hirano, N. 1999, ApJ, 525, L105

Zinchenko, I., Henning, Th., \& Schreyer, K. 1996, A\&AS, 124, 385

Zhou, S., Wu, U., Evans, N. J. II, Fuller, G. A., \& Myers, P. C. 1989, ApJ, 346, 168

Zhou, S. 1995, ApJ, 445, 685 